\title{
Increased arginase activity contributes to airway remodelling in chronic allergic asthma
}

\author{
H. Maarsingh*, B.G.J. Dekkers*, A.B. Zuidhof*, I.S.T. Bos*, M.H. Menzen*, T. Klein*, \\ G. Flik ${ }^{\#}$, J. Zaagsma* and H. Meurs*
}

ABSTRACT: Airway remodelling, characterised by increased airway smooth muscle (ASM) mass, subepithelial fibrosis, goblet cell hyperplasia and mucus gland hypertrophy, is a feature of chronic asthma. Increased arginase activity could contribute to these features via increased formation of polyamines and L-proline downstream of the arginase product L-ornithine, and via reduced nitric oxide synthesis.

Using the specific arginase inhitibor 2(S)-amino-6-boronohexanoic acid (ABH), we studied the role of arginase in airway remodelling using a guinea pig model of chronic asthma. Ovalbuminsensitised guinea pigs were treated with ABH or PBS via inhalation before each of 12 weekly allergen or saline challenges, and indices of arginase activity, and airway remodelling, inflammation and responsiveness were studied $24 \mathrm{~h}$ after the final challenge.

Pulmonary arginase activity of repeatedly allergen-challenged guinea pigs was increased. Allergen challenge also increased ASM mass and maximal contraction of denuded tracheal rings, which were prevented by $\mathrm{ABH}$. $\mathrm{ABH}$ also attenuated allergen-induced pulmonary hydroxyproline (fibrosis) and putrescine, mucus gland hypertrophy, goblet cell hyperplasia, airway eosinophilia and interleukin-13, whereas an increased L-ornithine/L-citrulline ratio in the lung was normalised. Moreover, allergen-induced hyperresponsiveness of perfused tracheae was fully abrogated by $\mathrm{ABH}$.

These findings demonstrate that arginase is prominently involved in allergen-induced airway remodelling, inflammation and hyperresponsiveness in chronic asthma.

KEYWORDS: Airway hyperresponsiveness, airway remodelling, eosinophils, fibrosis, goblet cells, polyamines

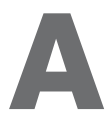
llergic asthma is a chronic inflammatory airway disease, which is characterised by allergen-induced, immunoglobulin (Ig)Emediated early and late bronchial obstructive reactions, acute and transient airway hyperresponsiveness (AHR) after these reactions and infiltration of inflammatory cells (particularly eosinophils and T-helper (Th) type 2 lymphocytes) into the airways [1, 2]. In addition, structural changes in the airway wall, including thickening of the basement membrane, increased airway smooth muscle (ASM) mass, subepithelial fibrosis, goblet cell hyperplasia, submucosal gland hypertrophy and increased vascularisation, are observed, which may contribute to the progressive decline in lung function and persistent AHR in chronic asthma $[2,3]$.
Recently, arginase, which hydrolyses L-arginine into L-ornithine and urea, has been identified as a key player in the pathophysiology of acute allergic asthma [4]. Two arginase isoforms have been identified, cytosolic arginase I and mitochondrial arginase II [5], both of which are expressed in the airways, particularly in epithelial cells, (myo)fibroblasts, endothelial cells and macrophages [6-8]. Increased expression and/or activity of arginase has been observed in airways and lung tissue obtained from various animal models of allergic asthma [9] and from patients [10-12]. Increased consumption of L-arginine by arginase contributes to the development of allergen-induced AHR in acute allergic asthma by limiting the bioavailability of L-arginine to nitric oxide synthase (NOS) isozymes [13-15],

\section{AFFLLIATIONS}

*Dept of Molecular Pharmacology, University of Groningen, and ${ }^{\#}$ Brains On-Line bv, Groningen, The Netherlands.

CORRESPONDENCE

H. Maarsingh

Dept of Molecular Pharmacology

University of Groningen

Antonius Deusinglaan 1

9713 AV Groningen

The Netherlands

E-mail: h.maarsingh@rug.nl

Received:

March 142010

Accepted after revision:

Jan 122011

First published online:

Feb 102011 
which hydrolyse the amino acid into nitric oxide and Lcitrulline. This leads to a deficiency of bronchodilating nitric oxide, and increased formation of the highly reactive proinflammatory and procontractile nitric oxide metabolite peroxynitrite, both of which underlie AHR in animal models of allergic asthma [13-18]. In a guinea pig model of allergic asthma, inhalation of the specific arginase inhibitor 2(S)amino-6-boronohexanoic acid $(\mathrm{ABH})$ acutely reversed allergeninduced AHR in vivo both after the early and late asthmatic reaction, to a similar extent as treatment with inhaled L-arginine [19]. Moreover, treatment with inhaled $\mathrm{ABH} 30 \mathrm{~min}$ prior to and $8 \mathrm{~h}$ after allergen challenge protected against the development of the early and late obstructive reactions, AHR after both reactions and airway inflammation [19].

Reduced nitric oxide synthesis due to increased arginase activity could also contribute to airway remodelling in chronic asthma, as nitric oxide inhibits ASM proliferation [20-23] and fibrosis [24]. In addition, increased arginase activity could induce airway remodelling via increased synthesis of polyamines (putrescine, spermidine and spermine) and L-proline downstream of L-ornithine $[5,9,25]$. Polyamines are involved in cell proliferation and differentiation [5] and increased polyamine levels have been demonstrated in allergen-challenged mice [10] and asthmatic patients [26]. L-Proline is a precursor of collagen [5] and could thus play a role in allergen-induced fibrosis. This is supported by the observation that inhibition of arginase activity reduced transforming growth factor (TGF)- $\beta$ induced collagen synthesis in primary mouse lung fibroblasts [27]. Therefore, we hypothesise that, as well as in acute asthma, increased arginase activity also plays a key role in the pathophysiology of chronic asthma. In support of this, increased arginase expression and activity have been observed in animal models of chronic allergic asthma [11, 28].

In the present study, we investigated the role of arginase in chronic asthma by studying the effects of inhaled $\mathrm{ABH}$ on allergen-induced airway remodelling, inflammation and AHR in repeatedly allergen-challenged guinea pigs.

\section{METHODS}

\section{Animals and sensitisation procedure}

Outbred, male, specified pathogen-free Dunkin Hartley guinea pigs (Harlan Hillcrest, Heathfield, UK) weighing 200-250 g were actively IgE-sensitised to ovalbumin (OVA), as described previously [29]. The animals were group-housed in individual cages in climate-controlled animal quarters, and given water and food ad libitum, while a 12-h on/12-h off light cycle was maintained.

All protocols described in this study were approved by the University of Groningen Committee for Animal Experimentation (Groningen, the Netherlands).

\section{Experimental protocol}

4 weeks after sensitisation, the animals were challenged once weekly with either saline (control group) or allergen (OVA) for 12 weeks. The saline and allergen provocations were performed by inhalation of aerosolised solutions, using a DeVilbiss nebuliser (type 646; DeVilbiss Healthcare, Somerset, PA, USA), in a specially designed animal cage, in which the guinea pigs could move freely [29]. OVA challenges were performed by inhalation of increasing concentrations of OVA in saline $(0.05$, $0.1,0.3,0.5$ and $0.7 \% \mathrm{w} / \mathrm{v}$ ) for $3 \mathrm{~min}$ each, with 7 -min intervals as previously described [29-31]. Allergen inhalations were discontinued when the first signs of respiratory distress were observed. No antihistaminic was needed to prevent the development of anaphylactic shock. Control animals were weekly challenged with saline for $3 \mathrm{~min}$.

The animals were treated with inhaled ABH in PBS $(25 \mathrm{mM}$ nebuliser concentration for $15 \mathrm{~min}$ ) or PBS (15 min) $0.5 \mathrm{~h}$ before and $8 \mathrm{~h}$ after each allergen or saline challenge. $\mathrm{ABH}$ was synthesised as described previously [32].

\section{Tissue acquisition}

At $24 \mathrm{~h}$ after the final challenge, guinea pigs were sacrificed by experimental concussion followed by rapid exsanguination. The lungs were immediately resected and kept on ice for further processing. The trachea was rapidly removed and transferred to a Krebs-Henseleit $(\mathrm{KH})$ solution $\left(37^{\circ} \mathrm{C}\right.$; $\left.\mathrm{pH} 7.4\right)$, pre-gassed with $5 \% \mathrm{CO}_{2} / 95 \% \mathrm{O}_{2}$.

\section{Isometric tension measurements}

Isometric contraction experiments were performed as described previously [30]. Briefly, the trachea was prepared such that it was free of serous connective tissue. Epithelium-denuded single open-ring preparations were mounted for isometric recording in organ baths containing $\mathrm{KH}$ solution $\left(37^{\circ} \mathrm{C}, \mathrm{pH} 7.4\right)$, gassed with $5 \% \mathrm{CO}_{2} / 95 \% \mathrm{O}_{2}$. After equilibration, the resting tension was adjusted to $0.5 \mathrm{~g}$, followed by pre-contractions using 20 and $40 \mathrm{mM} \mathrm{KCl}$. Following wash-outs and another equilibration period of $30 \mathrm{~min}$, cumulative concentration-response curves were constructed using methacholine.

\section{Tracheal perfusion}

The trachea was prepared such that it was free of serosal connective tissue and cut into two halves of $\sim 16 \mathrm{~mm}$ each. The preparations were attached to a perfusion holder and placed in an organ bath $\left(37^{\circ}\right)$ containing $20 \mathrm{~mL}$ gassed $\mathrm{KH}$ solution (extraluminal compartment) as described previously [17]. The tracheal lumen was perfused with recirculating $\mathrm{KH}$ solution from a separate 20-mL bath (intraluminal compartment) at constant flow rate, and hydrostatic pressure was measured at the proximal and distal ends of the trachea using axially centred side-hole catheters. The differential pressure $(\Delta P)$ was recorded; $\Delta P$ reflects the resistance of the tracheal segment to perfusion, which is a function of the mean diameter of the trachea between the pressure taps [33].

After a 45-min equilibration period with three washes with fresh $\mathrm{KH}$ (both intra- and extraluminal), $1 \mu \mathrm{M}$ isoprenaline was added to the extraluminal compartment to assess basal tone. After three washes (10 $\mathrm{min}$ each), the trachea was exposed to $40 \mathrm{mM} \mathrm{KCl}$ in $\mathrm{KH}$ (extraluminal) to obtain a receptor-independent reference response. After washout with $\mathrm{KH}$ for $45 \mathrm{~min}$, a cumulative concentration response curve was made with intraluminal methacholine. Intraluminal responses of the tracheal tube preparations to methacholine were expressed as a percentage of the response induced by extraluminal administration of $40 \mathrm{mM} \mathrm{KCl}$, to correct for differences in baseline $\Delta P$ and in $\Delta P$ changes in response to contractile stimuli, due to variation in internal diameter of the preparations used. 


\section{Arginase activity}

Arginase activity was determined in lung homogenates, by measuring the conversion of $\left[{ }^{14} \mathrm{C}\right]$-L-arginine to $\left[{ }^{14} \mathrm{C}\right]$-urea, as previously described [15]. Arginase activity was expressed as pmol urea produced per $\mathrm{mg}$ protein per min.

\section{Interleukin-13 measurement}

Levels of interleukin (IL)-13 were determined in lung homogenates by ELISA, using anti-guinea pig IL-13 (Cusabio, Newark, DE, USA) according to the manufacturer's protocol.

\section{Histochemistry}

Transverse cryostat cross sections $(4 \mu \mathrm{m})$ of the main bronchi from both the left and right lung lobes were used for morphometric analyses. Sections were stained for smooth muscle-specific myosin heavy chain (Neomarkers; Thermo Fisher, Fremont, CA, USA) and mucin 5 subtypes A and C (MUC5AC; Neomarkers). Primary antibodies were visualised using horseradish peroxidase-linked secondary antibodies and diaminobenzidine. In addition, haematoxylin-stained nuclei within the ASM bundle were counted. Eosinophils were identified in haematoxylin- and eosin-stained lung sections, and mucus-producing cells were stained using periodic acidSchiff (PAS). Airways within sections were digitally photographed and subclassified as cartilaginous or noncartilaginous. All immunohistochemical measurements were carried out digitally using quantification software (ImageJ; National Institutes of Health, Bethesda, MD, USA). For all studied parameters, three to six airways of each classification were analysed per animal.

\section{Analysis of amino acids and putrescine}

Lung homogenates $(50 \mu \mathrm{L})$ were precipitated by adding equal volumes of cold acetonitrile. After centrifugation $(5 \mathrm{~min}$ at $16,000 \times g), 30 \mu \mathrm{L}$ of the clear supernatant was mixed with $4 \mu \mathrm{L}$ deuterated internal standard solution $\left(10^{-5} \mathrm{M}\right)$, and used directly for the analysis of the amino acids L-arginine, L-ornithine and L-citrulline, and of putrescine.

Quantitative analysis was carried out by liquid chromatography-mass spectrometry using an LC-20 chromatography platform (Shimadzu, Kyoto, Japan) coupled to an API-4000 triple-quadrupole mass spectrometer with Turbo spray electrospray ionisation interface (AB Sciex, Concord, ON, Canada). Samples were derivatised with $40 \mu \mathrm{L}$ SymDAQ reagent (Brainlink, Groningen, the Netherlands) immediately prior to analysis using automated reagent addition on a SIL-10 autosampler (Shimadzu), and resolved on a $100 \times 3-\mathrm{mm}$ Synergi Max-RP (2.5- $\mu \mathrm{m}$ particle size) column (Phenomenex, Torrance, CA, USA) with a linear gradient of acetonitrile in water, each containing $0.1 \%(\mathrm{v} / \mathrm{v})$ formic acid. Eight-point calibration curves $(0.1-50 \mu \mathrm{M}$ for putrescine and $0.01-25 \mu \mathrm{M}$ for amino acids) were used for quantitation. The concentrations of the amino acids and putrescine in the lung homogenates were expressed as micromoles per milligram of protein.

\section{Hydroxyproline assay}

The hydroxyproline content of lung tissue was determined to estimate collagen content, as decribed previously [34]. The lower right lung lobe was pulverised under liquid nitrogen and sonicated in $10 \mathrm{~mL}$ PBS. Homogenates were incubated on ice with $1.25 \mathrm{~mL} \mathrm{5 \%} \mathrm{trichloroacetic} \mathrm{acid} \mathrm{for} 20 \mathrm{~min}$. Samples were centrifuged and the pellet was resuspended in $10 \mathrm{~mL}$ $12 \mathrm{~N}$ hydrochloric acid and heated at $110^{\circ} \mathrm{C}$ until charred and dry. The dried pellets were dissolved in $2 \mathrm{~mL}$ ultrapure water by incubating at room temperature for $72 \mathrm{~h}$ with intermittent vortexing. $5 \mu \mathrm{L}$ of each sample was transferred to a 96-well plate and $100 \mu \mathrm{L}$ chloramine-T solution $(1.4 \%$ chloramine- $\mathrm{T}$ in $0.5 \mathrm{M}$ sodium acetate $/ 10 \%$ isopropanol) was added. After $30 \mathrm{~min}, 100 \mu \mathrm{L}$ Ehrlich's solution (1.0 M 4-dimethylaminobenzaldehyde in $70 \%$ isopropanol $/ 30 \%$ perchloric acid) was added and samples were incubated at $65^{\circ} \mathrm{C}$ for $30 \mathrm{~min}$. Samples were cooled to room temperature and the amount of hydroxyproline was quantified by colorimetric measurement (optical density at $550 \mathrm{~nm}$; Biorad 680 plate reader, Biorad, Veenendaal, the Netherlands).

\section{Data analysis}

Both for the isometric contraction and the perfusion experiments, the maximal contractile response to methacholine was defined as maximal effect (Emax). Using this Emax, the sensitivity (negative logarithm of the effective concentration to cause $50 \%$ of the maximal contractile response (pEC50)) towards methacholine was evaluated.
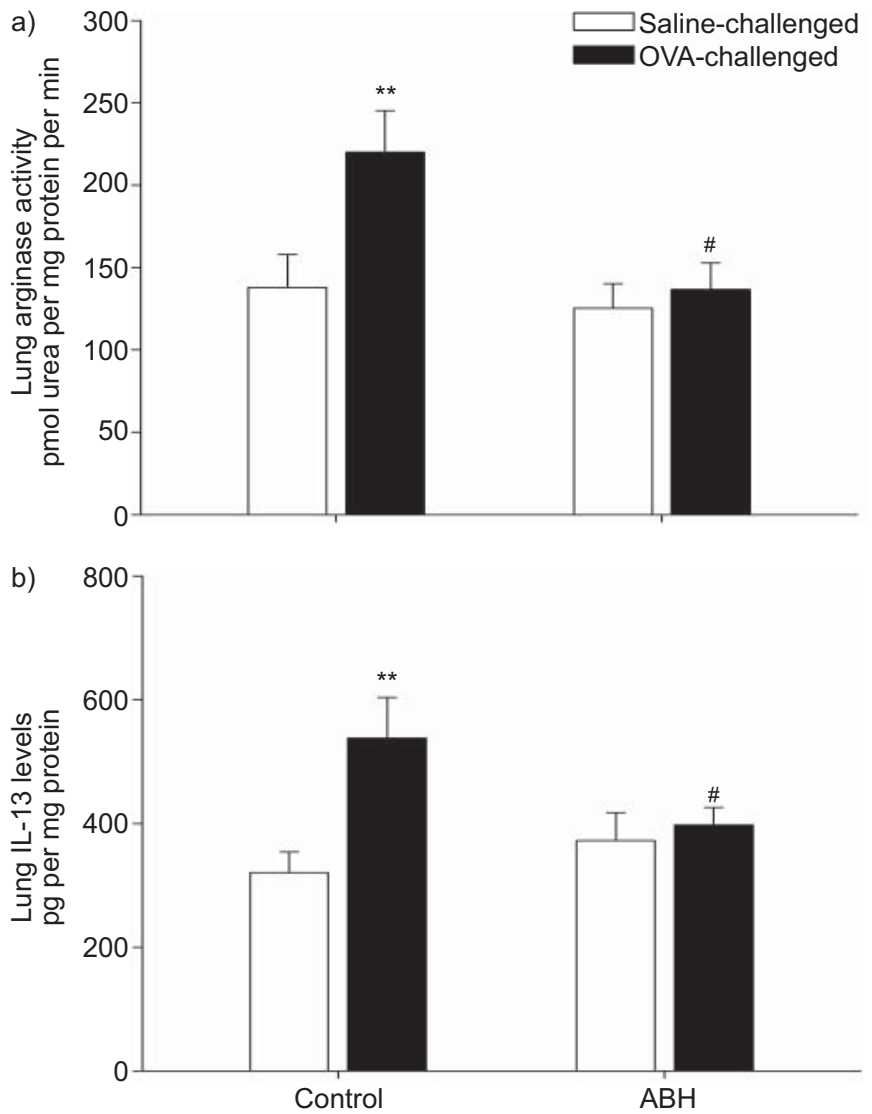

FIGURE 1. a) Arginase activity and b) interleukin (IL)-13 levels in lung homogenates from repeatedly saline- and allergen (ovalbumin (OVA))-challenged guinea pigs, treated with inhaled PBS (control) or 2(S)-amino-6-boronohexanoic acid $(\mathrm{ABH})$. Data are presented as mean \pm SEM of 7-8 experiments. ${ }^{*}: \mathrm{p}<0.05$ compared with OVA-challenged controls; **: $\mathrm{p}<0.01$ compared with saline-challenged controls. 


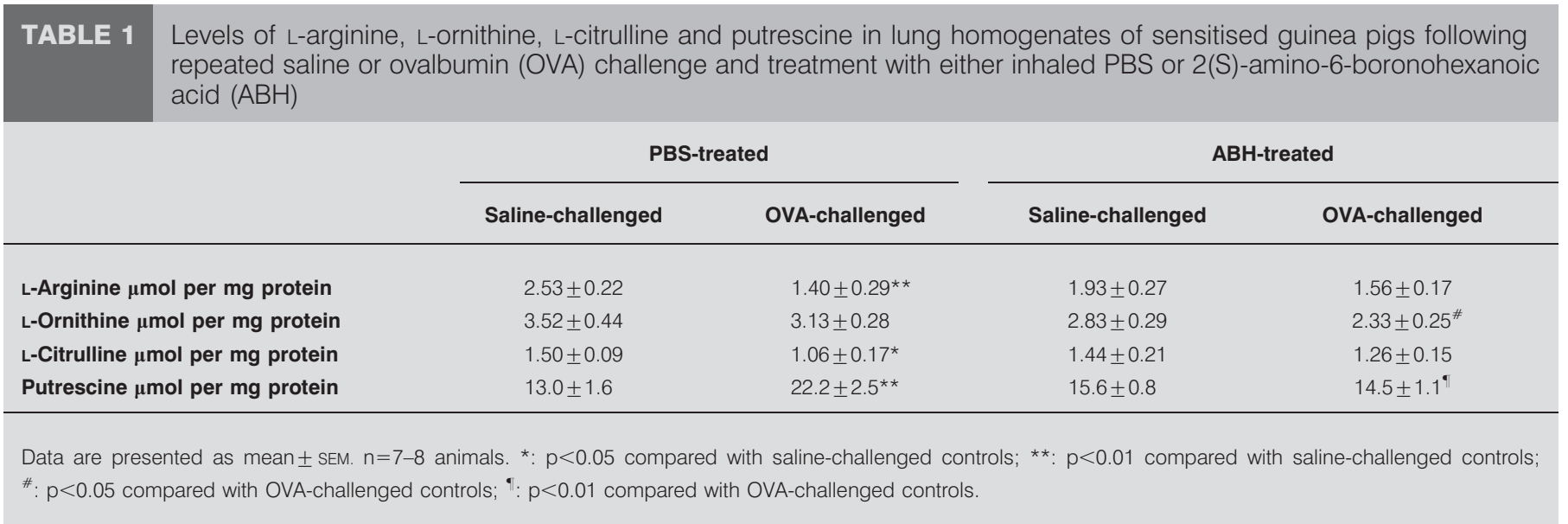

All data represent mean \pm SEM from $n$ separate experiments. Statistical significance of differences was evaluated using oneway ANOVA, followed by a Bonferoni post hoc test. Differences were considered statistically significant at $p<0.05$.

\section{RESULTS}

The arginase activity in lung homogenates from repeatedly allergen-challenged guinea pigs was 1.6-fold increased compared
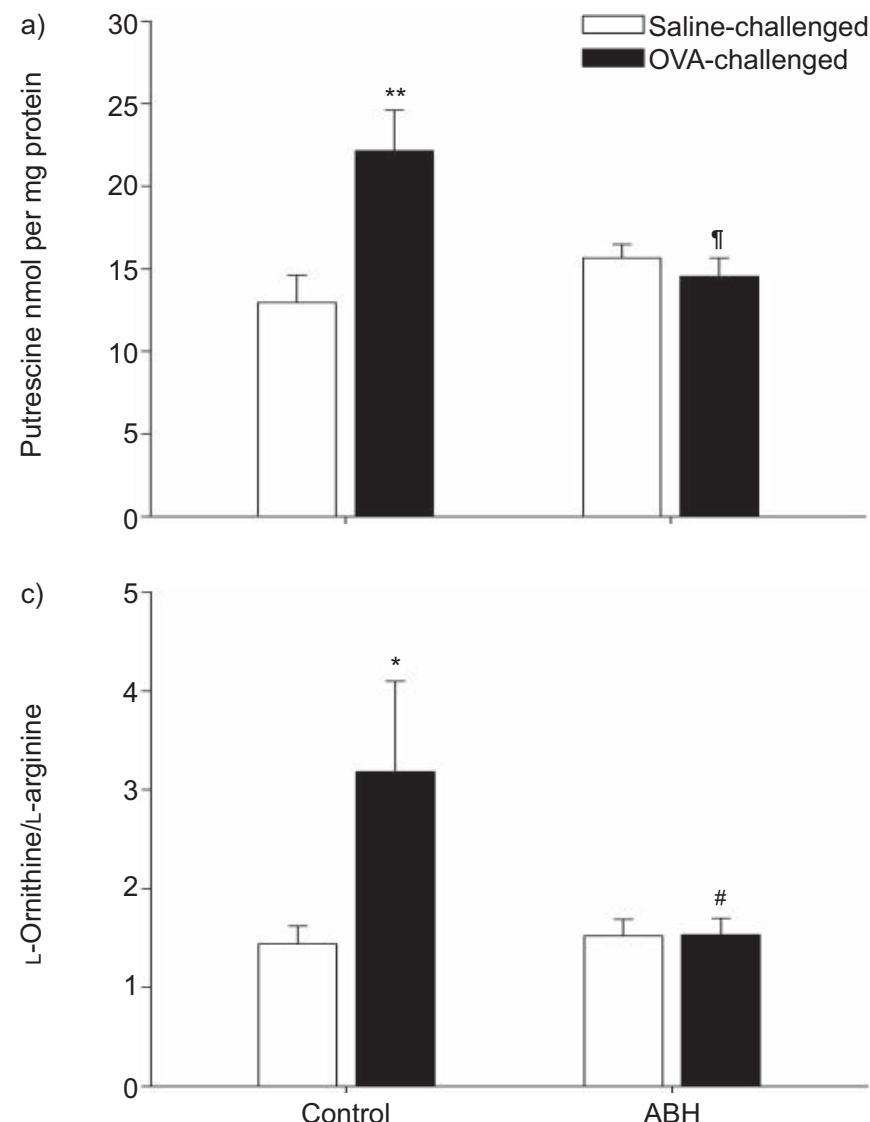

with saline-challenged controls ( $\mathrm{p}<0.01$; fig. 1a). In vivo treatment with inhaled $\mathrm{ABH}$ completely prevented the increased arginase activity in the multiple-challenged animals $(p<0.05)$, but did not affect arginase activity in the saline-challenged controls (fig. 1a).

In order to gain insight into the possible mechanisms underlying these changes in arginase activity, we determined lung levels of IL-13, a Th2 cytokine known to induce arginase
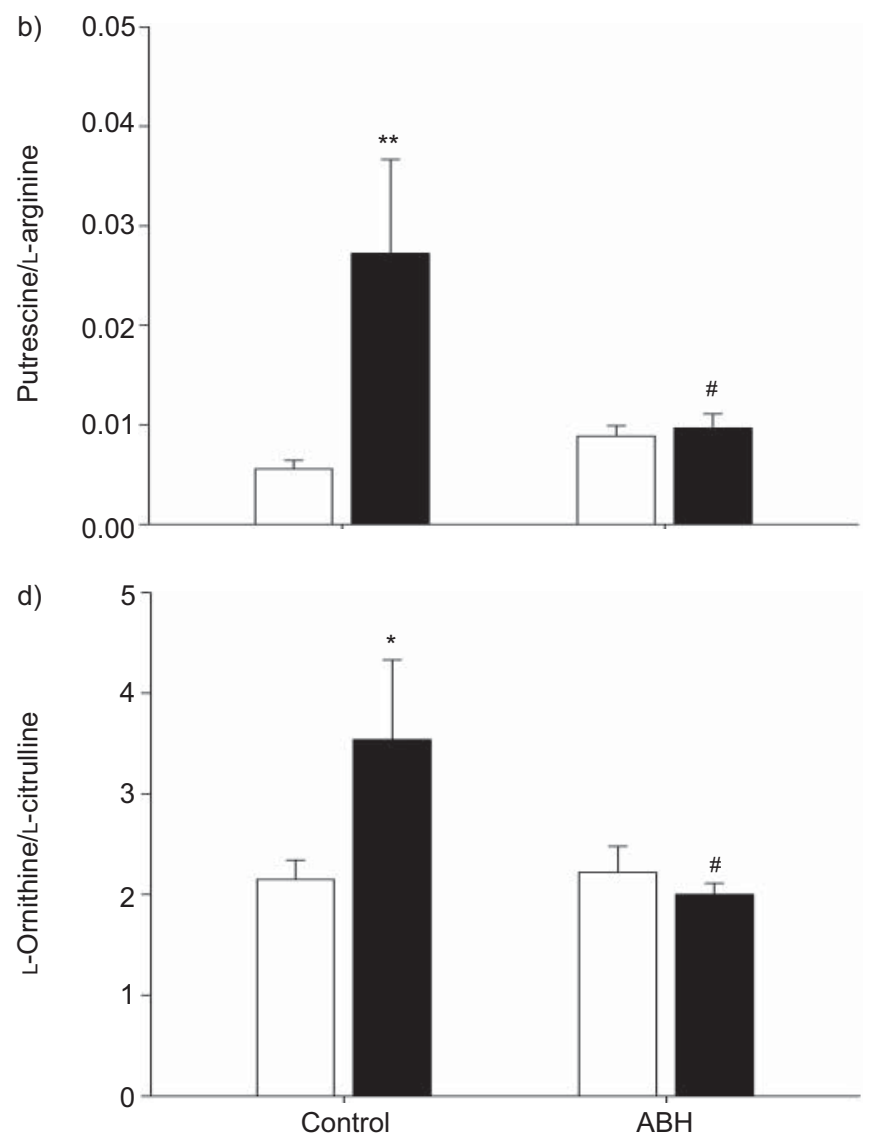

FIGURE 2. a) Putrescine levels, and b) putrescine/L-arginine, c) L-ornithine/L-arginine and d) L-ornithine/L-citrulline ratios in lung homogenates from repeatedly salineand allergen (ovalbumin (OVA))-challenged guinea pigs, treated with inhaled PBS (control) or 2(S)-amino-6-boronohexanoic acid (ABH). Data are presented as mean \pm SEM of 7-8 experiments. *: $p<0.05$ compared with saline-challenged controls; ${ }^{* *}: p<0.01$ compared with saline-challenged controls; ${ }^{*}: p<0.05$ compared with OVA-challenged controls; ": $p<0.01$ compared with OVA-challenged controls. 

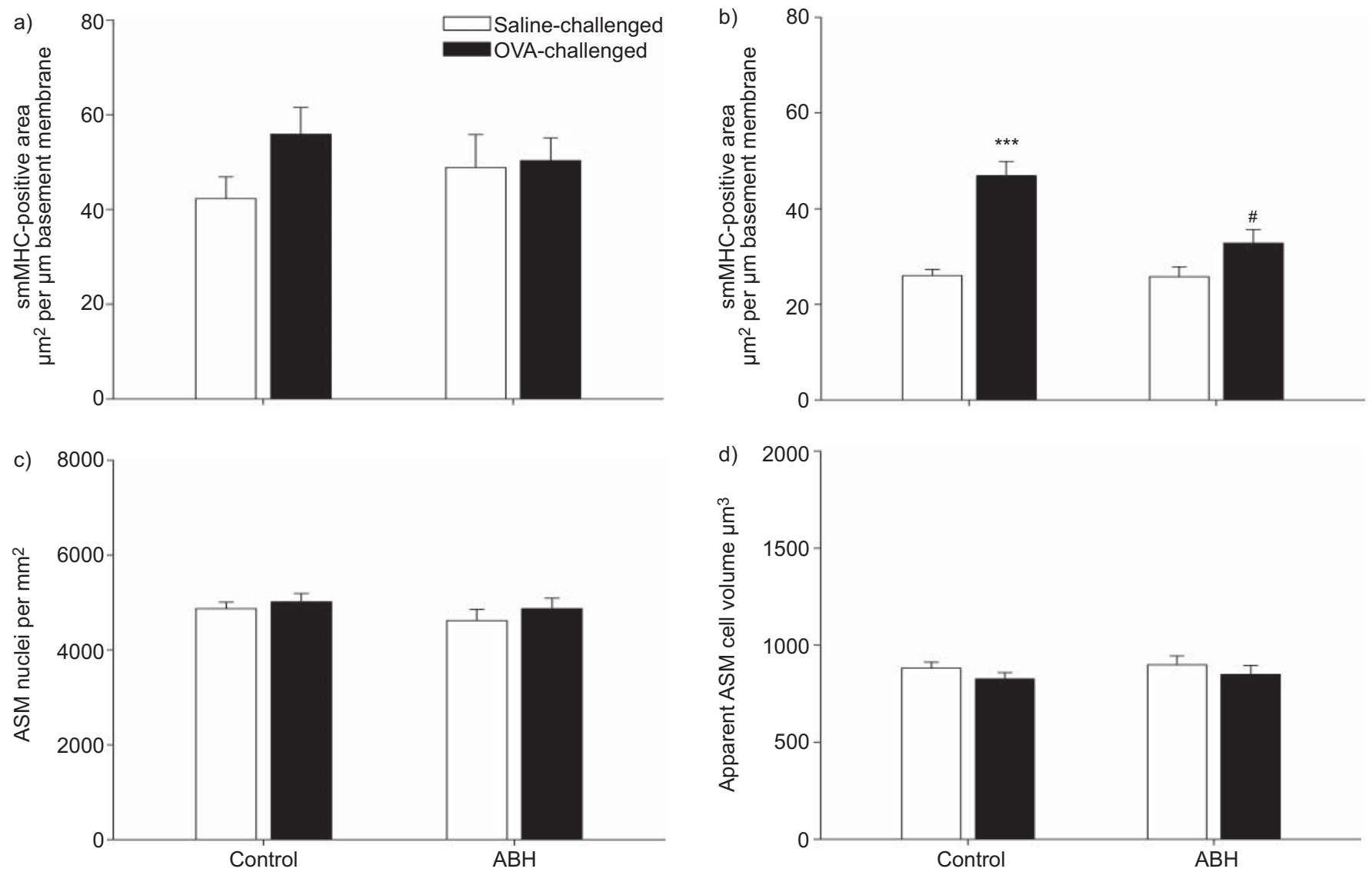

FIGURE 3. Airway smooth muscle (ASM) mass in a) cartilaginous and b) noncartilaginous airways from repeatedly saline- and allergen (ovalbumin (OVA))-challenged guinea pigs, treated with inhaled PBS (control) or 2(S)-amino-6-boronohexanoic acid (ABH). c) Number of nuclei per ASM area and d) apparent ASM cell volume in noncartilaginous airways from above-mentioned animals. Data are presented as mean \pm SEM of 7-8 experiments. smMHC: smooth muscle myosin heavy chain. ***: $p<0.001$ compared with saline-challenged controls; ${ }^{*}$ : $p<0.001$ compared with OVA-challenged controls.

activity in the lung [35]. As shown in figure 1b, IL-13 levels in the lung paralleled the changes in arginase activity. Thus, chronic allergen challenge resulted in a 1.7-fold increase in IL13 compared with saline-challenged controls ( $<<0.01$; fig. $1 b$ ). Treatment with $\mathrm{ABH}$ normalised the allergen-induced increase in IL-13 $(\mathrm{p}<0.05)$, leaving basal IL-13 levels unaffected (fig. 1b).

Pulmonary L-arginine and L-citrulline levels were decreased after repeated allergen challenge $(p<0.01$ and $p<0.05$, respectively; table 1), whereas L-ornithine was not significantly changed and the polyamine putrescine was increased $(\mathrm{p}<0.01$; table 1 and fig. $2 \mathrm{a})$. Accordingly, the putrescine/ L-arginine, L-ornithine/L-arginine and L-ornithine/L-citrulline ratios were significantly increased after the allergen challenges (fig. $2 b-d$ ), the L-ornithine/L-citrulline ratio indicating that increased arginase activity in the lung competes with NOS activity and reduces NO production. Consistent with this, all allergen-induced changes were attenuated by $\mathrm{ABH}$ (fig. 2 and table 1). ABH did not affect amino acid and polyamine levels in the saline-challenged animals (fig. 2 and table 1).

Repeated allergen challenge resulted in a trend towards an increased ASM mass in the cartilaginous airways compared with saline-challenged controls $(p=0.08$; fig. $3 a)$, whereas a marked increase in ASM mass was observed in the noncartilaginous airways $(p<0.001$; fig. $3 b)$. This increase was largely prevented by treatment with inhaled $\mathrm{ABH}(\mathrm{p}<0.001$; fig. 3b). In contrast, treatment with $\mathrm{ABH}$ did not affect ASM mass in the saline-challenged animals. In order to determine whether allergen-induced changes in ASM content were associated with changes in cell number and/or changes in cell size, the numbers of nuclei within the ASM layer were counted and expressed relative to total ASM area. From these data and the thickness of all sections, the apparent cell volume of the ASM cells was also calculated. OVA challenge did not change the number of nuclei per square millimetre of smooth muscle area (fig. 3c) or ASM cell volume (fig. 3d), indicating that the cell size is unchanged and the allergen-induced increase in ASM mass is caused by an increased cell number. ASM cell size was not affected by treatment with $\mathrm{ABH}$ either (fig. $3 \mathrm{c}$ and $3 \mathrm{~d}$ ).

Consistent with previous studies [30, 31], repeated allergen challenge induced a 1.7-fold increase in maximal contraction of epithelium-denuded tracheal open-ring preparations to methacholine compared with saline-challenged controls $(\mathrm{p}<0.01$; fig. $4 \mathrm{a}$ and table S1 in the online supplementary material), without an effect on the sensitivity (pEC50) towards the agonist. This indicates the development of a hypercontractile ASM phenotype, due to changes distal to the receptor, as 

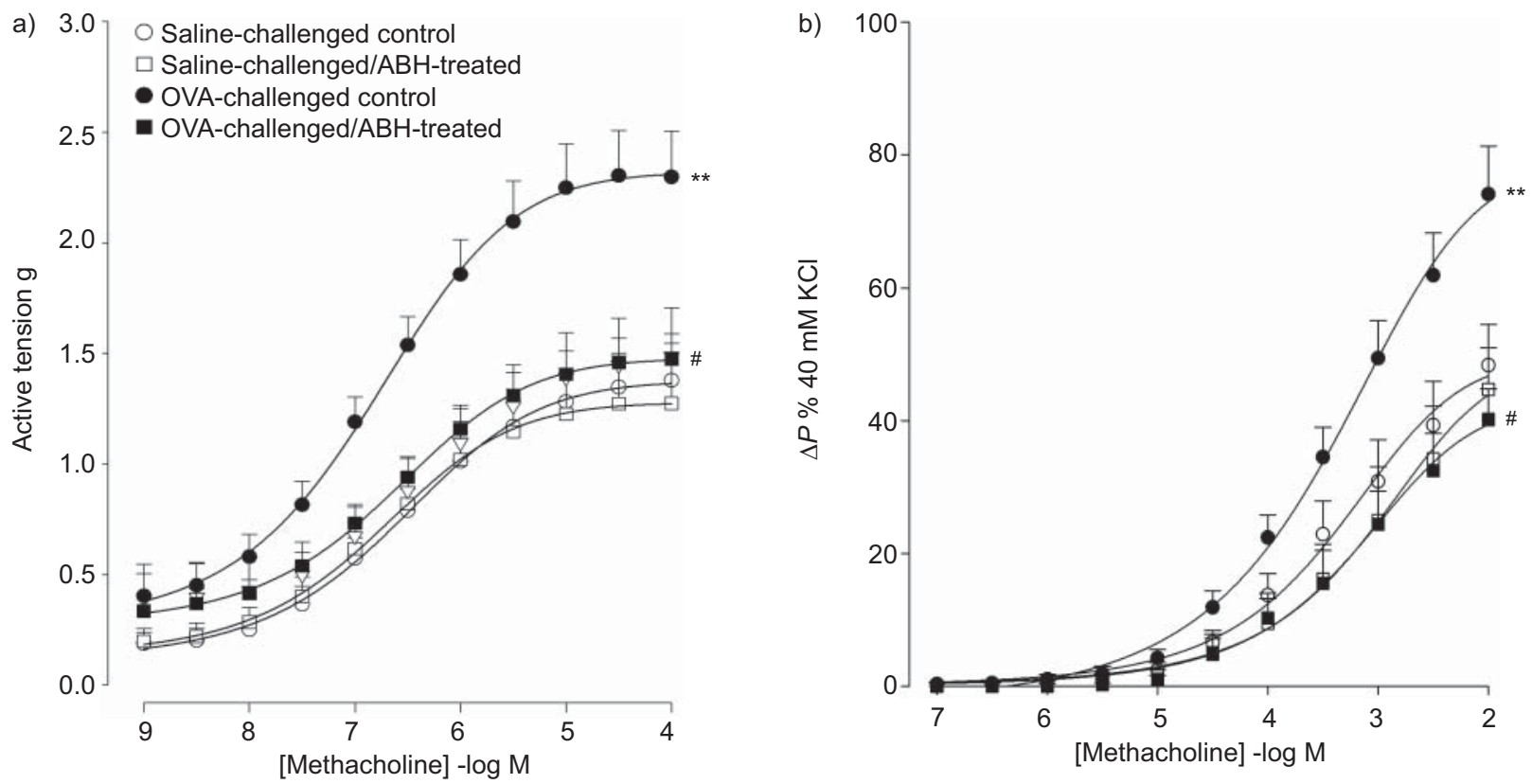

FIGURE 4. a) Methacholine-induced isometric contraction of epithelium-denuded tracheal open-ring preparations and b) constriction of intact perfused guinea pig tracheae from repeatedly saline- and allergen (ovalbumin (OVA))-challenged guinea pigs, treated with inhaled PBS (control) or 2(S)-amino-6-boronohexanoic acid (ABH). Data are presented as mean \pm SEM of a) 6-8 or b) 6-10 experiments. $\Delta P$ : differential pressure. ${ }^{* *}: p<0.01$ compared with saline-challenged controls; ${ }^{*}: p<0.01$ compared with OVA-challenged controls.

described previously [30,31]. Treatment with ABH completely prevented the allergen-induced hypercontractility $(\mathrm{p}<0.01)$, whereas ASM contractility of the saline-challenged animals was not affected (fig. $4 \mathrm{a}$ and table S1 in the online supplementary material).

In addition to intrinsic changes in the ASM, allergen challenge may also affect regulatory processes in the airway wall that determine ASM tone, including (epithelial) nitric oxide homeostasis $[16,17,28]$. Therefore, we also determined the responsiveness of intact perfused tracheal preparations to methacholine. The responsiveness of these preparations is expressed proportional to

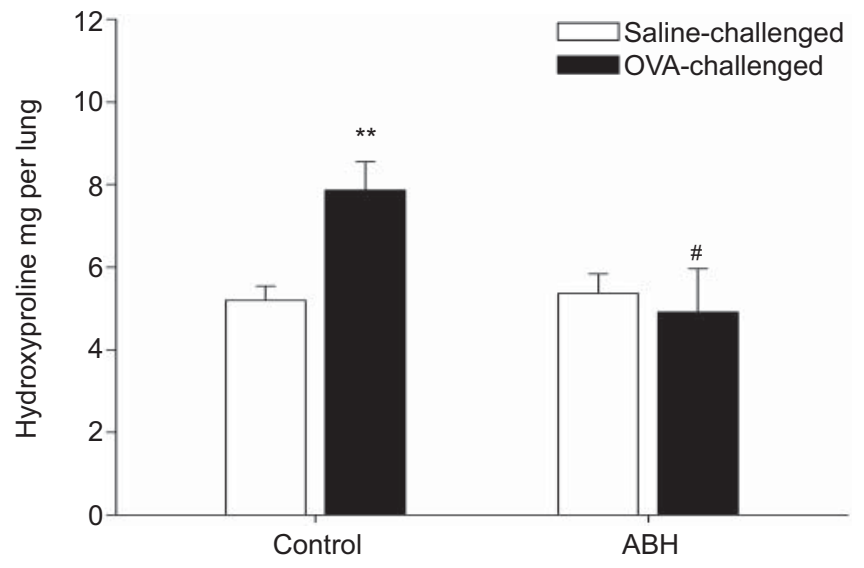

FIGURE 5. Effect of PBS (control) or 2(S)-amino-6-boronohexanoic acid (ABH) treatment in vivo on lung hydroxyproline content from multiple saline- or allergen (ovalbumin (OVA))-challenged guinea pigs. Data are presented as mean \pm SEM of 4-6 experiments. ${ }^{*}: p<0.05$ compared with OVA-challenged controls; **: $p<0.01$ compared with saline-challenged controls.
$\mathrm{KCl}$-induced airway constriction and, therefore, the induction of a hypercontractile ASM phenotype due to post-receptor changes, in the denuded rings, does not influence this airway responsiveness. Interestingly, a 1.7-fold increase in Emax was observed in the perfused tracheal segments obtained from allergen-challenged guinea pigs compared with those from saline-challenged controls $(p<0.01)$, without an effect on the sensitivity to the contractile agonist (fig. $4 \mathrm{~b}$ and table S2 in the online supplementary material). The development of AHR in these preparations was prevented by $\mathrm{ABH}$ treatment of the allergenchallenged animals $(p<0.01)$, whereas ABH did not affect the airway responsiveness of saline-challenged animals (fig. $4 \mathrm{~b}$ and table S2 in the online supplementary material).

Increased deposition of extracellular matrix proteins, including collagens, in the airway wall is a characteristic feature of airway remodelling in chronic asthma $[36,37]$. Since detection of collagens in the guinea pig airways by immunohistochemistry was not feasible with the antibodies available, lungs were analysed for hydroxyproline content, as an estimate of total collagen. Repeated allergen challenge caused a 1.5-fold increase in hydroxyproline $(p<0.01)$, which was fully prevented in the ABH-treated animals $(p<0.05)$, whereas $A B H$ did not affect hydroxyproline content in saline-challenged animals (fig. 5).

In the saline-treated animals, repeated allergen challenge induced eosinophil influx in the submucosal, ASM and adventitial compartments of both cartilaginous and noncartilaginous airways compared with saline-challenged controls (all $\mathrm{p}<0.001$; table 2). $\mathrm{ABH}$ treatment did not affect airway eosinophil numbers in the saline-challenged animals, but largely prevented the allergen-induced eosinophilia in all compartments of both the cartilaginous and noncartilaginous airways (all $\mathrm{p}<0.001$; table 2 ). 
Consistent with a previous study using the same guinea pig model of chronic asthma [31], repeated allergen challenge resulted in goblet cell hyperplasia in the cartilaginous airways $(\mathrm{p}<0.01$; fig. $6 \mathrm{a}$ and $\mathrm{b})$, which was attenuated by the arginase inhibitor $(p<0.05)$. In addition, repeated allergen challenge resulted in an increase in mucus gland area in the same airways ( $<<0.01$; fig. $6 \mathrm{c}$ ). This mucus gland hypertrophy was largely prevented by treatment with $\mathrm{ABH}$ ( $\mathrm{p}<0.01$; fig. 6c). Similarly, $\mathrm{ABH}$ attenuated the allergen-induced increase in MUC5AC-positive goblet cells $(p<0.05$; fig. $6 \mathrm{~d})$. Treatment with $\mathrm{ABH}$ did not affect numbers of mucus-producing cells in saline-challenged animals (fig. 6).

\section{DISCUSSION}

Using a well-defined guinea pig model of chronic allergic asthma, we have demonstrated that increased arginase activity contributes to allergen-induced airway remodelling, inflammation and AHR. Thus, inhalation of the arginase inhibitor $\mathrm{ABH}$ caused considerable protection against allergen-induced ASM hyperplasia and hypercontractility, mucus gland hypertrophy, goblet cell hyperplasia and fibrosis. In addition, ex vivo AHR to methacholine and airway eosinophilia induced by repeated allergen challenge were also greatly reduced in the ABH-treated animals.

In our guinea pig model, repeated allergen challenge resulted in a 1.6-fold increase in arginase activity in the lung, which is consistent with a recent finding in a mouse model of chronic asthma [11]. Remarkably, in vivo treatment with $\mathrm{ABH}$ completely prevented the allergen-induced increase in arginase activity. The changes in arginase activity were reflected by changes in the L-ornithine/L-arginine and putrescine/ $\mathrm{L}$-arginine ratios in the lung. Since $\mathrm{ABH}$ treatment did not affect the arginase activity in the saline-challenged animals, its effect cannot be ascribed to residual $\mathrm{ABH}$ in the lung, suggesting that arginase inhibition prevents induction of the enzyme after allergen challenge. The expression of arginase is strongly induced by Th2 cytokines, including IL-13 [35]; ABH markedly attenuated the allergen-induced increase in IL-13 in our model, indicating that the increased arginase activity and the inhibitory effect of $\mathrm{ABH}$ thereon may result from changes in IL-13. This is consistent with a recent finding in a mouse model of acute asthma, showing that intranasal application of the arginase inhibitor $\mathrm{N}^{\omega}$-hydroxy-nor-arginine (nor-NOHA) prevents house dust mite-induced increases in Th2 cytokine expression and arginase activity in the lung [38]. It is tempting to speculate that the previously observed anti-allergic effect of inhaled $\mathrm{ABH}$ [19] underlies the reduction of Th2 cytokine release and subsequent arginase induction.

Increased ASM mass is a characteristic feature of airway remodelling in asthma, which may result both from ASM cell hyperplasia and hypertrophy [39]. Consistent with previous studies [30, 31], repeated allergen challenge induced an increased ASM mass in our model, particularly in the noncartilaginous airways, characterised by ASM cell hyperplasia. Treatment with $\mathrm{ABH}$ inhibited the allergen-induced ASM cell hyperplasia. This could involve restoration of allergen-induced nitric oxide deficiency, as nitric oxide inhibits mitogen-induced proliferation of cultured human [20-22] and guinea pig [23] ASM cells. Accordingly, we demonstrated that the L-ornithine/L-citrulline ratio in lung tissue was significantly increased after repeated allergen challenge and normalised by the $\mathrm{ABH}$ treatment. A second mechanism by which arginase inhibition may inhibit ASM hyperplasia is via inhibition of L-ornithine production. L-Ornithine is a precursor of polyamines (putrescine, spermidine and spermine), which are involved in cell proliferation $[5,9,25,40]$. In support of this, transfection with arginase I increased polyamine levels and cell proliferation in vascular smooth muscle cells [41]. Both arginase and ornithine decarboxylase (ODC), which converts L-ornithine into putrescine, are expressed in airway epithelial cells [42] and induction of both enzymes by growth factors, leading to increased polyamine levels, has been described [4]. Moreover, elevated polyamine levels have been detected in the lungs of allergen-challenged mice [10] and in the serum of asthmatic patients [26]. We now demonstrate that the allergeninduced increase in arginase activity causes an increased

TABLE 2 Infiltration of eosinophils into different compartments of the airway wall of sensitised guinea pigs following repeated saline or ovalbumin (OVA) challenge, and treatment with either inhaled PBS or 2(S)-amino-6-boronohexanoic acid (ABH)

Airway compartment

Eosinophils cells per $\mathrm{mm}$ basement membrane

\begin{tabular}{|c|c|c|c|c|}
\hline & \\
\hline & \multicolumn{2}{|c|}{ PBS-treated } & \multicolumn{2}{|c|}{ ABH-treated } \\
\hline & Saline-challenged & OVA-challenged & Saline-challenged & OVA-challenged \\
\hline \multicolumn{5}{|l|}{ Cartilaginous } \\
\hline Submucosa & $10.7 \pm 1.0$ & $50.1 \pm 5.1^{\star \star \star}$ & $12.9 \pm 3.3$ & $22.4 \pm 3.0^{*, \#}$ \\
\hline ASM & $0.1 \pm 0.0$ & $1.2 \pm 0.2^{\star \star \star}$ & $0.2 \pm 0.1$ & $0.6 \pm 0.1^{*, \#}$ \\
\hline Adventitia & $14.9 \pm 1.9$ & $63.2 \pm 6.7^{\star \star \star}$ & $20.3 \pm 3.4$ & $27.7 \pm 2.6^{\#}$ \\
\hline \multicolumn{5}{|c|}{ Noncartilaginous } \\
\hline Submucosa & $7.6 \pm 0.8$ & $43.2 \pm 3.5^{\star \star \star}$ & $12.4 \pm 3.9$ & $21.9 \pm 3.6^{*, \#}$ \\
\hline ASM & $0.1 \pm 0.1$ & $1.5 \pm 0.3^{\star \star *}$ & $0.2 \pm 0.1$ & $0.7 \pm 0.2^{*, \#}$ \\
\hline Adventitia & $26.3 \pm 3.1$ & $113.3 \pm 9.9^{\star \star \star}$ & $25.3 \pm 3.6$ & $62.4 \pm 6.8^{* * *}, \#$ \\
\hline
\end{tabular}

Data are presented as mean \pm SEM. $n=7-8$ animals. ASM: airway smooth muscle. ${ }^{*}: \mathrm{p}<0.05$ compared with saline-challenged controls; ${ }^{\star} \star \star$ : $\mathrm{p}<0.001$ compared with saline-challenged controls; ${ }^{*}: \mathrm{p}<0.001$ compared with OVA-challenged controls. 

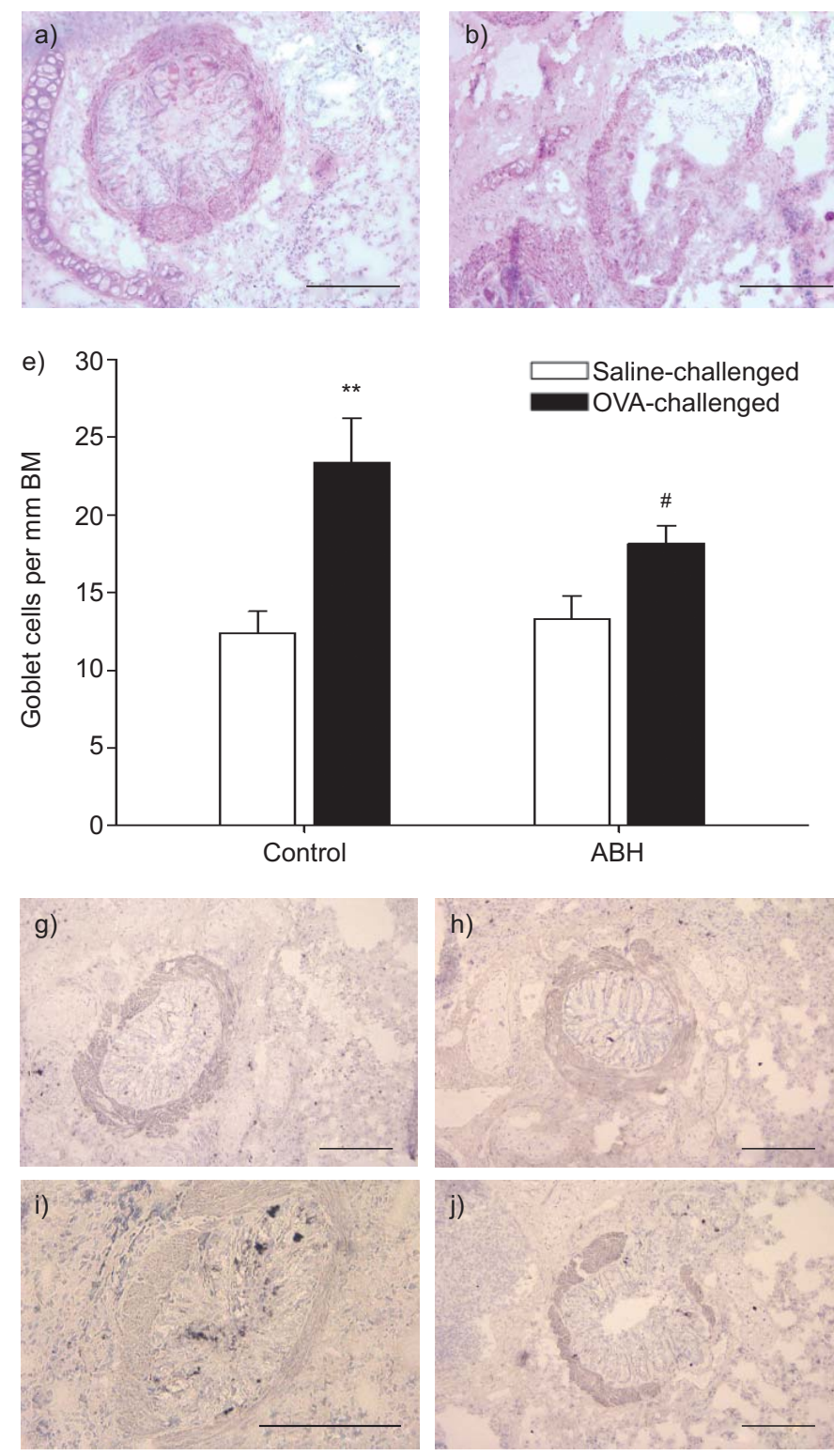
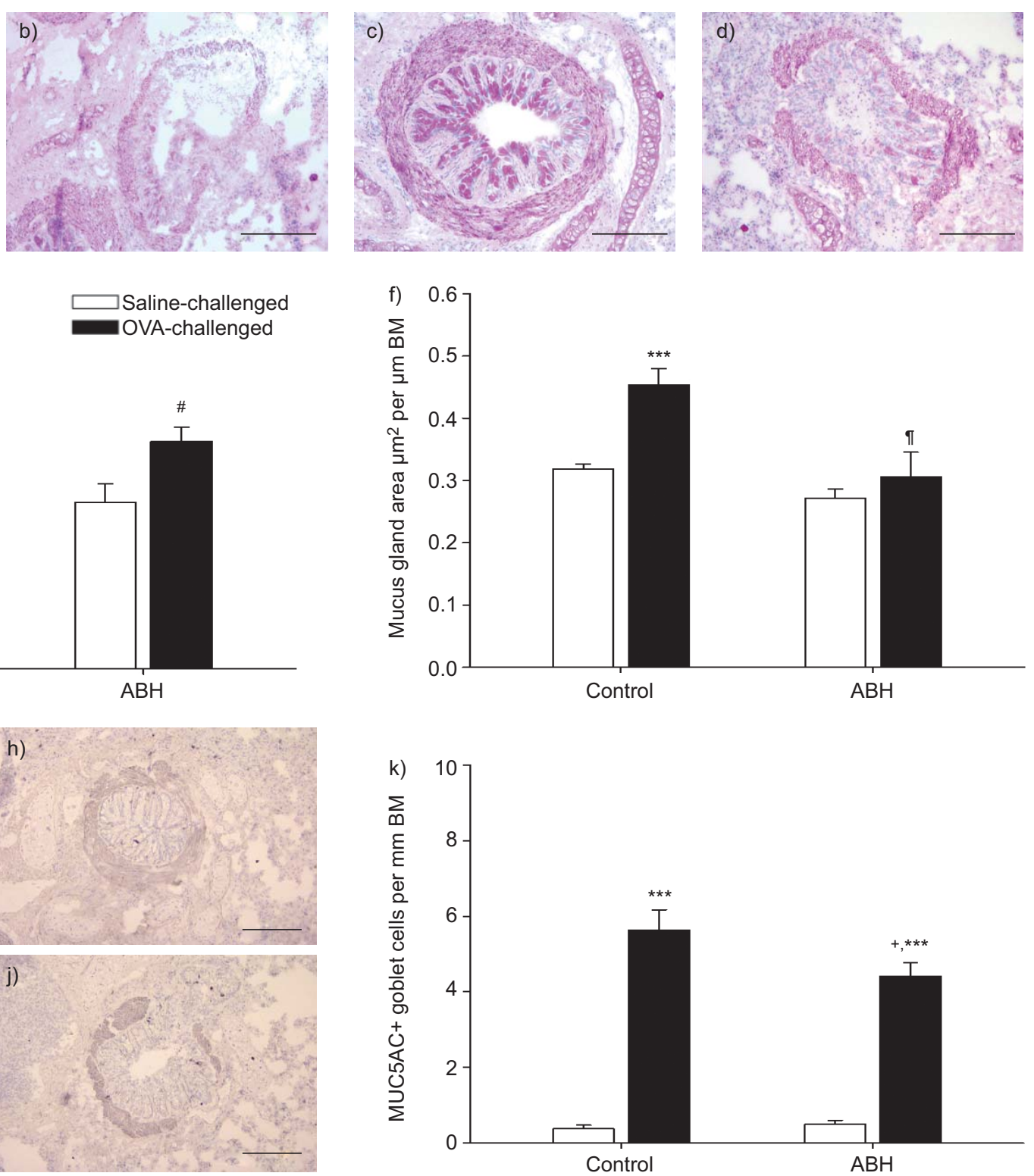

FIGURE 6. Periodic acid-Schiff (PAS) staining of intrapulmonary cartilaginous airway sections from a) saline-challenged, PBS-treated, b) saline-challenged, 2(S)amino-6-boronohexanoic acid (ABH)-treated, c) ovalbumin (OVA)-challenged, PBS-treated, and d) OVA-challenged, ABH-treated guinea pigs. Effects of in vivo treatment with PBS (control) or ABH on e) goblet cell number and f) mucus gland area. Mucin 5 subtypes $\mathrm{A}$ and $\mathrm{C}$ (MUC5AC) staining of intrapulmonary cartilaginous airway sections from g) saline-challenged, PBS-treated, h) saline-challenged, ABH-treated, i) OVA-challenged, PBS-treated and j) OVA-challenged, ABH-treated guinea pigs. k) MUC5AC-positive goblet cell numbers in intrapulmonary cartilaginous airways from saline- or OVA-challenged guinea pigs. Representative examples of PAS and MUC5AC stainings are shown in panels $\mathrm{a}-\mathrm{d}$ ) and $\mathrm{g}-\mathrm{j}$ ), respectively. Scale bars $=100 \mu \mathrm{m}$. Data are presented as mean \pm SEM of 7-8 experiments. BM: basement membrane. ${ }^{+}: p<0.05 \mathrm{compared}$ with $\mathrm{OVA}-$ challenged controls; ${ }^{*}: \mathrm{p}<0.01$ compared with saline-challenged controls; ${ }^{* *}: \mathrm{p}<0.001$ compared with saline-challenged controls; ${ }^{*}: p<0.01$ compared with OVAchallenged controls; " : $p<0.001$ compared with OVA-challenged controls.

production of the polyamine putrescine, which is completely abolished by $\mathrm{ABH}$. In addition to increased formation of L-ornithine, a deficiency of nitric oxide may also contribute to the increased polyamine levels after repeated allergen challenge, as nitric oxide inhibits ODC via S-nitrosylation [43].

Repeated allergen challenge also increased maximal contraction of tracheal single-ring preparations to methacholine. Previous experiments using the same animal model have demonstrated that this ASM hypercontractility results from increased expression of contractile proteins [31]. Interestingly, our findings indicate that increased arginase activity contributes to the induction of a hypercontractile ASM phenotype. This could be caused by an increased formation of peroxynitrite following multiple allergen challenges [28], as the expression of contractile proteins in human fetal lung fibroblasts is increased by this nitrogen species [44]. It should be noticed that, in addition to a hypercontractile ASM phenotype, allergeninduced changes in stimuli governing ASM tone, including nitric oxide, may lead to the induction of AHR in chronic asthma 
[28]. Thus, previous experiments using the same guinea pig model demonstrated that repeated allergen challenge induced hyperresponsiveness of perfused tracheal segments to methacholine, which was caused by increased synthesis of peroxynitrite due to uncoupling of inducible NOS (iNOS), as a consequence of increased L-arginine consumption by arginase [28]. The present finding suggests that in vivo treatment of repeatedly allergen-challenged guinea pigs with $\mathrm{ABH}$ protects against the uncoupling of iNOS, and subsequent peroxynitrite formation and AHR.

Airway fibrosis in chronic asthma could also result from increased arginase activity, via increased production of L-proline downstream of L-ornithine. In support of this, the profibrotic factor TGF- $\beta$ has been shown to induce arginase activity in mouse lung and fibroblasts [45]. Moreover, increased expression of arginases I and II was closely associated with increased collagen I and hydroxyproline expression in bleomycin-induced lung fibrosis in mice [27, 46]. Using inhaled $A B H$, we now demonstrate that increased arginase activity contributes to allergen-induced fibrosis as well. Similarly, the antifibrotic effect of $\mathrm{ABH}$ could result from inhibition of the arginaseinduced deficiency in nitric oxide, which has an antifibrotic action [24]. Accordingly, allergen-induced collagen deposition was shown to be higher in lungs from iNOS ${ }^{-/-}$mice and mice chronically treated with the NOS inhibitor $\mathrm{L}-\mathrm{N}^{\mathrm{G}}$-nitroarginine methyl ester [47, 48].

Another characteristic feature of chronic allergic asthma is airway inflammation, which presumably plays an important role in airway remodelling [2]. Our current study indicates that increased arginase activity may be involved in this process, since the infiltration of eosinophils into different compartments of the airway wall following repeated allergen challenge was markedly reduced by $\mathrm{ABH}$ treatment. This anti-inflammatory effect of $\mathrm{ABH}$ corresponds to previous observations in a guinea pig model of acute asthma [19], and could be explained by increased production of nitric oxide and reduced formation of proinflammatory peroxynitrite. Thus, allergeninduced inflammatory responses are attenuated in mice that overexpress endothelial NOS [49] and are elevated in iNOS ${ }^{-/-}$ mice [50] compared with wild-type mice. Consistent with our observations, treatment with the arginase inhibitor nor-NOHA reduced inflammatory cell numbers in the bronchoalveolar lavage (BAL) of allergen-challenged mice [38, 50]. However, the arginase inhibitor S-(2-boronoethyl)-L-cysteine did not affect inflammatory cell numbers or cytokine levels in BAL, and even further enhanced peribronchiolar and perivascular inflammation in another mouse model of acute allergic asthma [51]. Remarkably, arginase I from bone marrow-derived cells appeared not to be required for allergen-induced recruitment of inflammatory cells into the lung [52], which could indicate a role for arginase in structural cells, particularly the airway epithelium, in this process.

Increased mucus secretion, which may result from mucus gland hypertrophy as well as goblet cell hyperplasia, is yet another feature of airway obstruction in chronic asthma [53]. We demonstrated that increased arginase activity also regulates changes in mucus cells and MUC5AC expression in repeatedly allergen-challenged guinea pigs. The increased arginase activity may contribute to these features via increased formation of polyamines, which induce cell proliferation and differentiation [5]. Interestingly, L-arginine treatment enhanced allergen-induced goblet hyperplasia in mice [54], which could be explained by increased L-ornithine synthesis from L-arginine by arginase. The anti-inflammatory and anti-allergic effects of $\mathrm{ABH}$ [19] could also affect mucus hypersecretion, as many inflammatory cytokines and mediators, including IL-13, are involved in mucus secretion, goblet cell hyperplasia and mucin expression in the airways [53]. In addition, nitric oxide synthesis has been shown to reduce mucus hypersecretion [55], whereas peroxynitrite strongly induces MUC5AC expression [56].

In conclusion, our findings demonstrate that increased arginase activity has a key role in allergen-induced airway remodelling, inflammation and AHR in chronic asthma. Treatment with inhaled ABH effectively inhibits these features, indicating that arginase inhibitors have therapeutic potential in the treatment of this disease.

\section{SUPPORT STATEMENT}

This study was financially supported by Merck Sharpe and Dohme, Oss, the Netherlands

\section{STATEMENT OF INTEREST}

Statements of interest for J. Zaagsma and H. Meurs, and for the study itself can be found at www.erj.ersjournals.com $/ \mathrm{site} / \mathrm{misc} /$ statements. xhtml

\section{REFERENCES}

1 Bousquet J, Jeffery PK, Busse WW, et al. Asthma. From bronchoconstriction to airways inflammation and remodeling. Am J Respir Crit Care Med 2000; 161: 1720-1745.

2 Cockcroft DW, Davis BE. Mechanisms of airway hyperresponsiveness. J Allergy Clin Immunol 2006; 118: 551-559.

3 Meurs H, Gosens R, Zaagsma J. Airway hyperresponsiveness in asthma: lessons from in vitro model systems and animal models. Eur Respir J 2008; 32: 487-502.

4 Maarsingh H, Zaagsma J, Meurs H. Arginase: a key enzyme in the pathophysiology of allergic asthma opening novel therapeutic perspectives. Br J Pharmacol 2009; 158: 652-664.

$5 \mathrm{Wu} \mathrm{G}$, Morris SM. Arginine metabolism: nitric oxide and beyond. Biochem J 1998; 336: 1-17.

6 Que LG, Kantrow SP, Jenkinson CP, et al. Induction of arginase isoforms in the lung during hyperoxia. Am J Physiol 1998; 275: L96-L102.

7 Klasen S, Hammermann R, Fuhrmann M, et al. Glucocorticoids inhibit lipopolysaccharide-induced up-regulation of arginase in rat alveolar macrophages. Br J Pharmacol 2001; 132: 1349-1357.

8 Lindemann D, Racke K. Glucocorticoid inhibition of interleukin-4 (IL-4) and interleukin-13 (IL-13) induced up-regulation of arginase in rat airway fibroblasts. Naunyn Schmiedebergs Arch Pharmacol 2003; 368: 546-550.

9 Maarsingh H, Pera T, Meurs H. Arginase and pulmonary diseases. Naunyn Schmiedebergs Arch Pharmacol 2008; 378: 171-184.

10 Zimmermann $\mathrm{N}$, King NE, Laporte $\mathrm{J}$, et al. Dissection of experimental asthma with DNA microarray analysis identifies arginase in asthma pathogenesis. J Clin Invest 2003; 111: 1863-1874.

11 North ML, Khanna N, Marsden PA, et al. Functionally important role for arginase 1 in the airway hyperresponsiveness of asthma. Am J Physiol Lung Cell Mol Physiol 2009; 296: L911-L920.

12 Morris CR, Poljakovic M, Lavrisha L, et al. Decreased arginine bioavailability and increased serum arginase activity in asthma. Am J Respir Crit Care Med 2004; 170: 148-153. 
13 Meurs H, McKay S, Maarsingh $\mathrm{H}$, et al. Increased arginase activity underlies allergen-induced deficiency of cNOS-derived nitric oxide and airway hyperresponsiveness. Br J Pharmacol 2002; 136: 391-398.

14 Maarsingh H, Leusink J, Bos IST, et al. Arginase strongly impairs neuronal nitric oxide-mediated airway smooth muscle relaxation in allergic asthma. Respir Res 2006; 7: 6 .

15 Maarsingh H, Bossenga BE, Bos IST, et al. L-Arginine deficiency causes airway hyperresponsiveness after the late asthmatic reaction. Eur Respir J 2009; 34: 191-199.

16 De Boer J, Meurs H, Flendrig L, et al. Role of nitric oxide and superoxide in allergen-induced airway hyperreactivity after the late asthmatic reaction in guinea-pigs. Br J Pharmacol 2001; 133 1235-1242.

17 De Boer J, Meurs H, Coers W, et al. Deficiency of nitric oxide in allergen-induced airway hyperreactivity to contractile agonists after the early asthmatic reaction: an ex vivo study. $\mathrm{Br} J$ Pharmacol 1996; 119: 1109-1116.

18 Schuiling M, Zuidhof $\mathrm{AB}$, Bonouvrie MA, et al. Role of nitric oxide in the development and partial reversal of allergen-induced airway hyperreactivity in conscious, unrestrained guinea-pigs. $\mathrm{Br}$ J Pharmacol 1998; 123: 1450-1456.

19 Maarsingh $\mathrm{H}$, Zuidhof $\mathrm{AB}$, Bos IS, et al. Arginase inhibition protects against allergic airway obstruction, hyperresponsiveness and inflammation. Am J Respir Crit Care Med 2008; 178: 565-573.

20 Hamad AM, Johnson SR, Knox AJ. Antiproliferative effects of NO and ANP in cultured human airway smooth muscle. Am J Physiol 1999; 277: L910-L918.

21 Hamad AM, Knox AJ. Mechanisms mediating the antiproliferative effects of nitric oxide in cultured human airway smooth muscle cells. FEBS Lett 2001; 506: 91-96.

22 Patel HJ, Belvisi MG, Donnelly LE, et al. Constitutive expressions of type I NOS in human airway smooth muscle cells: evidence for an antiproliferative role. FASEB J 1999; 13: 1810-1816.

23 Kizawa Y, Ohuchi N, Saito K, et al. Effects of endothelin-1 and nitric oxide on proliferation of cultured guinea pig bronchial smooth muscle cells. Comp Biochem Physiol C Toxicol Pharmacol 2001; 128: 495-501.

24 Wani J, Carl M, Henger A, et al. Nitric oxide modulates expression of extracellular matrix genes linked to fibrosis in kidney mesangial cells. Biol Chem 2007; 388: 497-506.

25 Meurs H, Maarsingh H, Zaagsma J. Arginase and asthma: novel insights into nitric oxide homeostasis and airway hyperresponsiveness. Trends Pharmacol Sci 2003; 24: 450-455.

26 Kurosawa M, Shimizu Y, Tsukagoshi H, et al. Elevated levels of peripheral-blood, naturally occurring aliphatic polyamines in bronchial asthmatic patients with active symptoms. Allergy 1992; 47: 638-643.

27 Kitowska K, Zakrzewicz D, Konigshoff M, et al. Functional role and species-specific contribution of arginases in pulmonary fibrosis. Am J Physiol Lung Cell Mol Physiol 2007; 294: L34-L45.

28 Maarsingh H, Bos IST, Westerhof-Humblot FJ, et al. Increased arginase activity underlies airway hyperresponsiveness in a guinea pig model of chronic allergic asthma. Am J Respir Crit Care Med 2007; 175: A522.

29 Meurs H, Santing RE, Remie R, et al. A guinea pig model of acute and chronic asthma using permanently instrumented and unrestrained animals. Nat Protoc 2006; 1: 840-847.

30 Gosens R, Bos IS, Zaagsma J, et al. Protective effects of tiotropium bromide in the progression of airway smooth muscle remodeling. Am J Respir Crit Care Med 2005; 171: 1096-1102.

31 Bos IS, Gosens R, Zuidhof AB, et al. Inhibition of allergen-induced airway remodelling by tiotropium and budesonide: a comparison. Eur Respir J 2007; 30: 653-661.

$32 \mathrm{Xu} \mathrm{L}$, Hilliard B, Carmody RJ, et al. Arginase and autoimmune inflammation in the central nervous system. Immunology 2003; 110: 141-148.
33 Munakata M, Mitzner W, Menkes H. Osmotic stimuli induce epithelial-dependent relaxation in guinea pig trachea. J Appl Physiol 1988; 64: 466-471.

34 Dekkers BG, Bos IS, Gosens R, et al. The integrin-blocking peptide RGDS inhibits airway smooth muscle remodeling in a guinea pig model of allergic asthma. Am J Respir Crit Care Med 2010; 181: 556-565.

35 Yang M, Rangasamy D, Matthaei KI, et al. Inhibition of arginase I activity by RNA interference attenuates IL-13-induced airways hyperresponsiveness. J Immunol 2006; 177: 5595-5603.

36 Jeffery PK. Remodeling in asthma and chronic obstructive lung disease. Am J Respir Crit Care Med 2001; 164: S28-S38.

37 Postma DS, Timens W. Remodeling in asthma and chronic obstructive pulmonary disease. Proc Am Thorac Soc 2006; 3: 434-439.

38 Takahashi N, Ogino K, Takemoto K, et al. Direct inhibition of arginase attenuated airway allergic reactions and inflammation in a Dermatophagoides farinae-induced NC/Nga mouse model. Am J Physiol Lung Cell Mol Physiol 2010; 299: L17-L24.

39 Dekkers BG, Maarsingh H, Meurs H, et al. Airway structural components drive airway smooth muscle remodeling in asthma. Proc Am Thorac Soc 2009; 6: 683-692.

40 Hoet PH, Nemery B. Polyamines in the lung: polyamine uptake and polyamine-linked pathological or toxicological conditions. Am J Physiol Lung Cell Mol Physiol 2000; 278: L417-L433.

41 Wei LH, Wu G, Morris SM Jr, et al. Elevated arginase I expression in rat aortic smooth muscle cells increases cell proliferation. Proc Natl Acad Sci USA 2001; 98: 9260-9264.

42 Bergeron C, Boulet LP, Page N, et al. Influence of cigarette smoke on the arginine pathway in asthmatic airways: increased expression of arginase I. J Allergy Clin Immunol 2007; 119: 391-397.

43 Bauer PM, Fukuto JM, Buga GM, et al. Nitric oxide inhibits ornithine decarboxylase by S-nitrosylation. Biochem Biophys Res Commun 1999; 262: 355-358.

44 Ichikawa $\mathrm{T}$, Sugiura $\mathrm{H}$, Koarai A, et al. Peroxynitrite augments fibroblast-mediated tissue remodeling via myofibroblast differentiation. Am J Physiol Lung Cell Mol Physiol 2008; 295: L800-L808.

45 Liu H, Drew P, Gaugler AC, et al. Pirfenidone inhibits lung allograft fibrosis through L-arginine-arginase pathway. Am J Transplant 2005; 5: 1256-1263.

46 Endo M, Oyadomari S, Terasaki Y, et al. Induction of arginase I and II in bleomycin-induced fibrosis of mouse lung. Am J Physiol Lung Cell Mol Physiol 2003; 285: L313-L321.

47 Prado CM, Leick-Maldonado EA, Kasahara DI, et al. Effects of acute and chronic nitric oxide inhibition in an experimental model of chronic pulmonary allergic inflammation in guinea pigs. Am J Physiol Lung Cell Mol Physiol 2005; 289: L677-L683.

48 Kenyon NJ, Gohil K, Last JA. Susceptibility to ovalbumin-induced airway inflammation and fibrosis in inducible nitric oxide synthetase-deficient mice: mechanisms and consequences. Toxicol Appl Pharmacol 2003; 191: 2-11.

49 Ten Broeke R, De Crom R, Van Haperen R, et al. Overexpression of endothelial nitric oxide synthase suppresses features of allergic asthma in mice. Respir Res 2006; 7: 58.

50 Bratt JM, Franzi LM, Linderholm AL, et al. Arginase enzymes in isolated airways from normal and nitric oxide synthase 2-knockout mice exposed to ovalbumin. Toxicol Appl Pharmacol 2009; 234: 273-280.

51 Ckless K, Lampert A, Reiss J, et al. Inhibition of arginase activity enhances inflammation in mice with allergic airway disease, in association with increases in protein S-nitrosylation and tyrosine nitration. J Immunol 2008; 181: 4255-4264.

52 Niese KA, Collier AR, Hajek AR, et al. Bone marrow cell derived arginase I is the major source of allergen-induced lung arginase but is not required for airway hyperresponsiveness, remodeling and lung inflammatory responses in mice. BMC Immunol 2009; 10: 33 . 
53 Rogers DF. Physiology of airway mucus secretion and pathophysiology of hypersecretion. Respir Care 2007; 52: 1134-1146.

54 Takano H, Lim HB, Miyabara Y, et al. Oral administration of Larginine potentiates allergen-induced airway inflammation and expression of interleukin-5 in mice. J Pharmacol Exp Ther 1998; 286: 767-771.
55 Ramnarine SI, Khawaja AM, Barnes PJ, et al. Nitric oxide inhibition of basal and neurogenic mucus secretion in ferret trachea in vitro. Br J Pharmacol 1996; 118: 998-1002.

56 Fischer BM, Voynow JA. Neutrophil elastase induces MUC5AC gene expression in airway epithelium via a pathway involving reactive oxygen species. Am J Respir Cell Mol Biol 2002; 26: 447-452. 